\title{
Universiteit
}

Leiden

The Netherlands

\section{Compliance with mismatch repair testing in pT1 colorectal cancer diagnosed before the age of 70 years}

Ykema, B.L.M.; Nagtegaal, I.D.; Kuhlmann, K.; Berkel, A.M. van; Leerdam, M.E. van; Dutch T1 CRC Working Grp

\section{Citation}

Ykema, B. L. M., Nagtegaal, I. D., Kuhlmann, K., Berkel, A. M. van, \& Leerdam, M. E. van. (2021). Compliance with mismatch repair testing in pT1 colorectal cancer diagnosed before the age of 70 years. Virchows Archiv, 479, 451-457. doi:10.1007/s00428-021-03074-w

Version: Publisher's Version

License: $\quad$ Creative Commons CC BY 4.0 license

Downloaded from: https://hdl.handle.net/1887/3276800

Note: To cite this publication please use the final published version (if applicable). 


\title{
Compliance with mismatch repair testing in pT1 colorectal cancer diagnosed before the age of 70 years
}

\author{
Berbel L. M. Ykema ${ }^{1}$ (D) • Iris D. Nagtegaal ${ }^{2} \cdot$ Koert Kuhlmann $^{3} \cdot$ Annemarie M. van Berkel $^{4}$ • \\ Monique E. van Leerdam ${ }^{1,5} \cdot$ on behalf of the Dutch T1 CRC Working Group
}

Received: 20 January 2021 / Revised: 12 February 2021 / Accepted: 1 March 2021

(C) The Author(s), under exclusive licence to Springer-Verlag GmbH Germany, part of Springer Nature 2021

\begin{abstract}
Mismatch repair (MMR) testing is recommended in the Netherlands for all patients under 70 years of age with newly diagnosed colorectal cancer (CRC) in order to identify Lynch syndrome. T1 CRC can be removed by local excision or oncological surgical resection. We evaluated the frequency of MMR testing in pT1 lesions within the Dutch CRC screening cohort. pT1 CRC diagnosed within the Dutch population-based screening program from 2016-2018 were identified by the Dutch pathology registry (PALGA). Pathology reports were evaluated, including registration of MMR testing (by immunohistochemistry and/ or microsatellite instability PCR). Frequency of MMR testing was compared between pT1 tumors that were treated by local (endoscopic or transanal) excision and oncological surgical resections. A total of 3.692 pT1 CRCs were diagnosed (median age 63 years, $61.4 \%$ males). MMR testing was performed in $83 \%$ and uptake increased over time (71\% in 2016 to $92 \%$ in 2018 , $p<0.01)$. MMR testing was significantly more often performed in younger patients and in academic hospitals. When pT1 CRC was treated by oncological surgical resection $(n=1.132)$, MMR testing was performed in $89 \%$ of cases and was known prior to oncological resection in $51 \%$ of cases. MMR testing occurred significantly less often in case of local excision ( $80 \%$ of $n=2.560$ ) compared to oncological surgical resection $(p<0.01)$. MMR testing was performed in $83 \%$ of T1 CRCs and uptake increased over time. MMR testing was more frequently performed in pT1 CRC resected by oncological surgical resection compared with local excision.
\end{abstract}

Keywords Colorectal cancer $\cdot$ Lynch syndrome $\cdot$ Mismatch repair deficiency $\cdot$ Screening

\section{Introduction}

Lynch syndrome is detected in $3 \%$ of all newly diagnosed colorectal carcinomas (CRC) [1]. Identification of Lynch syndrome is relevant for both patients and their relatives, as

Monique E. van Leerdam

m.v.leerdam@nki.nl

1 Department of Gastroenterology and Hepatology, Netherlands Cancer Institute, Amsterdam, the Netherlands

2 Department of Pathology, Radboud University Medical Center, Nijmegen, The Netherlands

3 Department of Surgery, Netherlands Cancer Institute, Amsterdam, The Netherlands

4 Department of Gastroenterology and Hepatology, Noordwest Ziekenhuisgroep, Alkmaar, The Netherlands

5 Department of Gastroenterology and Hepatology, Leiden University Medical Center, Leiden, The Netherlands surveillance can be offered and subtotal colectomy instead of segmental resection can be advised in order to improve survival and reduce cancer incidence [2-5]. In the Netherlands, mismatch repair (MMR) deficiency testing has been recommended since January 2016 in all newly diagnosed CRC before the age of 70 years $[6,7]$.

T1 CRC differs from the other CRC stages as complete local (endoscopic or transanal) excision may be sufficient instead of oncological surgical resection. With the introduction of the national CRC screening program in 2014, there is a shift towards increased proportion of pT1 CRC in the Netherlands [8]. However, it is unknown whether adherence to MMR testing is different for pT1 CRC treated by local excision versus oncological surgical resection.

This study aims to determine the compliance to MMR testing in pT1 CRCs diagnosed within the Dutch populationbased screening program. Furthermore, we will evaluated whether differences in MMR deficiency testing occur between tumors removed by local (endoscopic or transanal) excision 
and surgical oncological surgical resection in academic and non-academic hospitals.

\section{Materials and methods}

\section{Study population}

In this retrospective study, patients with newly diagnosed CRC were identified through a search of the nationwide network and registry of histopathology and cytopathology in the Netherlands (PALGA), registered as LZV2020-44 [9]. Patients with CRC, diagnosed between 55 and 75 years of age within the Dutch population-based screening program, were selected $[10,11]$. CRC diagnosed before 2016 (guideline recommended MMR testing since 2016 in the Netherlands for all CRC under the age of 70 [12]), $\geq 70$ years of age at diagnosis and tumor stages pTis, pT2, pT3, and pT4 were excluded. Patients with well differentiated neuroendocrine tumors, neuroendocrine carcinoma, squamous cell carcinoma, or metastases in the colorectum were also excluded. The obtained pathology reports of each potential pT1 CRC case were reviewed. The pathology reports contained information about type of resection, $\mathrm{T}$ and $\mathrm{N}$ status, if available, and results of immunohistochemistry of MMR proteins (MLH1, MSH2, MSH6 and PMS2 by IHC), microsatellite instability testing (MSI) and $M L H 1$ promoter hypermethylation if performed [13].

Differences between local excision and segmentally resected pT1 CRC, type of laboratory (academic or nonacademic) and year of diagnosis were evaluated. Local excision included endoscopic excision and transanal endoscopic surgery. Oncological surgical resection was performed primarily or secondary after local excision. CRC in the proximal colon was defined as located between cecum and splenic flexure or distally located from descending colon to rectum.

\section{Statistical analysis}

SPSS Statistics (version 22) was used for statistical analysis. Data were summarized using descriptive statistics. Categorical and continuous data between groups were compared by chi-square tests and independent $t$-test, respectively. Values of $p<0.05$ were considered significant. All authors had access to the study data and reviewed and approved the final manuscript.

\section{Results}

\section{Patient characteristics}

A total of 3.692 pT1 CRCs were diagnosed within the Dutch CRC screening program between January 2016 and
December 2018 (Fig. 1). The median age at CRC diagnosis was 63 years (range 55-69 years) and the majority were male $(61 \%)$. Most CRC were located in the distal colon $(84 \%$, Table 1). pT1 CRC were removed by local excision in 2.560 $(69 \%)$ and oncological surgical resection in $1.132(31 \%)$ of the cases (Table 1). Oncological surgical resection was performed after a local excision in 291 of $1132(26 \%)$ of the pT1 CRC.

\section{MMR testing}

MMR testing was performed in $3.050(83 \%)$ of newly diagnosed pT1 CRC. MMR testing increased over time $(71 \%$ in 2016, 87\% in 2017 and $92 \%$ in 2018, $p<0.01)$. MMR testing was most frequently tested by IHC (71\% of 3.050 , Fig. 2$)$. The majority of IHC MMR testing occurred by staining for the four proteins $(2.792$ out of $2.842,98 \%)$. MSI PCR only was performed in $6 \%$ of cases.

In 207 cases, both IHC MMR and MSI PCR were performed on the same tumor material with a concordance of 97\%. The concordance was 99\% when MMR testing was performed on biopsy or local excision in comparison with oncological surgical resection material $(n=83)$.

Among the pT1 CRC treated by local excision only, MMR testing was performed in 2.048 cases $(80 \%)$ compared with 1.002 cases $(89 \%, p<0.01)$ treated by oncological surgical resection. Because MMR status could influence the type of surgery, we evaluated how often MMR status was known before oncological surgical resection. In 552 (51\%) patients that underwent oncological surgical resection, the MMR result was performed on biopsy or local excision samples and known prior to surgery. Over time the uptake of MMR testing increased from $67.3 \%$ to $90.6 \%$ for local excision $(p<0.01)$ and $79.1 \%$ to $96.7 \%$ for surgical resection ( $p<0.01$, Fig. 3 ).

MMR testing was more often performed in academic setting compared to non-academic setting $(90 \%$ vs $81 \%, p<0.01)$ and more often for patients at younger age (88\% vs $80 \%$, $p<0.01$, Table 2). MSI PCR only was performed in $1 \%$ in academic laboratories versus $8 \%$ of non-academic laboratories $(p$-value $<0.01)$.

\section{MMR deficiency}

MMR deficiency was detected in 186 of the 3.050 T1 CRCs of cases were MMR status was evaluated (6\%). Of those cases loss of MLH1 and/or PMS2 staining was found in $81 \%$. In cases with MLH1 deficiency, $M L H 1$ promoter hypermethylation status was evaluated in $133(89 \%)$ of the cases. In 15/150 cases with MLH1 deficiency both MLH1 promoter hypermethylation status and BRAF was evaluated. In 16/150 cases, neither $M L H 1$ promoter hypermethylation nor BRAF was determined. 
Fig. 1 Study flowchart

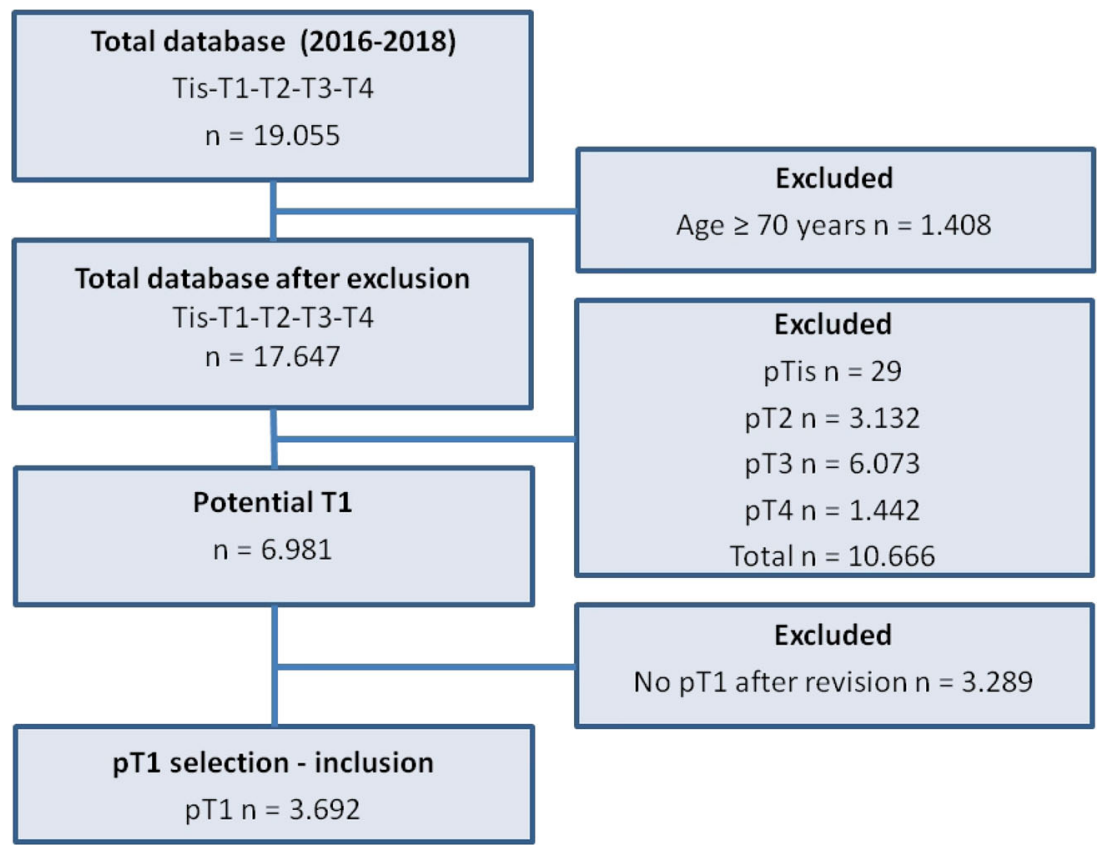

MMR deficient pT1 CRC were diagnosed more frequently in females, in the age category 61-69 years and were more

Table 1 Baseline characteristics of pT1 colorectal cancer (CRC)

\begin{tabular}{ll}
\hline Characteristic & $n=3.692$ \\
\hline Gender & \\
Male $(n, \%)$ & $2266(61 \%)$ \\
Female $(n, \%)$ & $1426(39 \%)$ \\
Age at diagnosis (median, min-max) & $63(55-69)$ \\
Year of diagnosis & \\
2016 & $1395(38 \%)$ \\
2017 & $1096(30 \%)$ \\
2018 & $1197(32 \%)$ \\
Location CRC & \\
Proximal $(n, \%)$ & $575(16 \%)$ \\
Distal $(n, \%)$ & $2995(84 \%)$ \\
Unknown & 122 \\
Type of carcinoma & \\
Adenocarcinoma $(n, \%)$ & $3589(97 \%)$ \\
Mucinous carcinoma $(n, \%)$ & $91(3 \%)$ \\
Signet cell carcinoma $(n, \%)$ & $11(0 \%)$ \\
Medullar carcinoma $(n, \%)$ & $1(0 \%)$ \\
Type of procedure & \\
Local excision $(n, \%)$ & $2560(69 \%)$ \\
Oncological surgical resection & $841(23 \%)$ \\
Local excision followed by oncological surgical resection & $291(8 \%)$ \\
Type of hospital & \\
Academic $(n, \%)$ ntd & $2990(81 \%)$ \\
Non-academic $(n, \%)$ & \\
\hline & \\
\hline &
\end{tabular}

*Proximal: cecum to splenic flexure frequently located proximal. MMR deficient tumors were more frequently removed by oncological surgical resection $(56 \%)$ compared with MMR proficient tumors $(31 \%$, $p<0.01$ ) (Table 3).

\section{Discussion}

The aim of this study was to evaluate the adherence to MMR testing in pT1 CRC diagnosed within the Dutch CRC screening program before the age of 70 years and whether differences in MMR testing exists between pT1 CRC after local excision and oncological surgical resection. We showed that MMR testing was performed in $83 \%$ of pT1 CRC cases diagnosed within the CRC screening program between 2016 and 2018, and testing increased over time. MMR testing was significantly more often performed after oncological surgical resection.

To reinforce the detection of Lynch syndrome, MMR testing has been recommended as routine diagnostics in newly diagnosed CRC diagnosed $<70$ years of age [12, 14-17]. MMR testing has been shown to be cost-effective when performed in all CRCs diagnosed before age of 70 years [18]. In one English hospital the compliance to MMR testing in newly diagnosed CRCs was 100\% [15], while in 19 Dutch hospitals compliance to MMR testing was $84 \%$ in all newly diagnosed CRCs including stage I to IV and diagnosed from January 2016 to July 2017 [19]. Our study evaluated specifically pT1 CRC. MMR testing is more conventionally performed on oncological surgical resection specimen, and therefore MMR testing may be forgotten when only local excision has 
Fig. 2 Mismatch repair (MMR) testing in pT1 colorectal carcinomas (CRC, $n=3.692)$

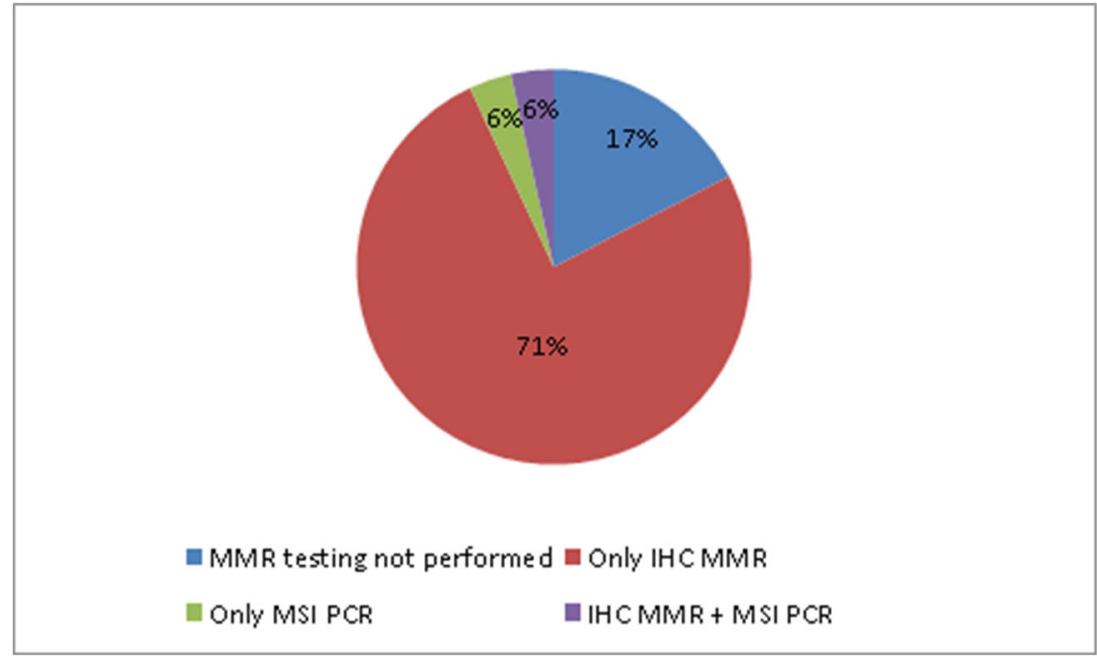

been performed. This may explain the relatively high rate of local excision specimen without MMR testing in up to $20 \%$.

In case of oncological surgical resection of pT1 CRC, MMR status was evaluated in $89 \%$ and was known prior to surgery in $51 \%$ of the cases. In a single-center UK study, MMR status was evaluated in $69 \%$ on diagnostic biopsies prior to surgery [15]. MMR testing should preferably be performed on biopsy material as the concordance of IHC between biopsies and resection specimen is high $[15,20,21]$. Furthermore, in case of Lynch syndrome the surgical procedure may be adapted: a subtotal colectomy instead of a segmental resection can be considered for patient diagnosed with $\mathrm{CRC}$ at a younger age in order to reduce the risk of a second primary CRC [22-24]. Implementation of testing on endoscopic material (biopsy or local excision specimen) would also improve the relatively low testing rate in pT1 CRC (currently $80 \%$ ).

In case local excision of pT1 CRC, MMR status was known in $80 \%$ of the cases. If local excision is feasible, routine biopsies are not advised because this may complicate the endoscopic mucosal resection $[6,7,25]$. In these patients, MMR status will be known after local excision.

The adherence to MMR testing guidelines increased over time for pT1 CRC, suggesting that the awareness increased after implementation of the guideline in 2016 in the Netherlands [12]. There was a clear difference in MMR testing between academic and non-academic hospitals (90\% vs. $81 \%$, respectively). Furthermore, MMR testing using MSI PCR only was performed more frequently in non-academic hospitals, lacking information about the loss of the specific protein detected by IHC. In the Netherlands, IHC MMR is the recommended test because of subsequent $M L H 1$ promoter hypermethylation or germline analyses to identify Lynch syndrome, and because of the easy implementation in the routine diagnostic workflow [12, 19].

Explanations for not performing MMR testing could be costs, delay in completion of pathology report, no quality control (some pathologist would be unaware of the updated

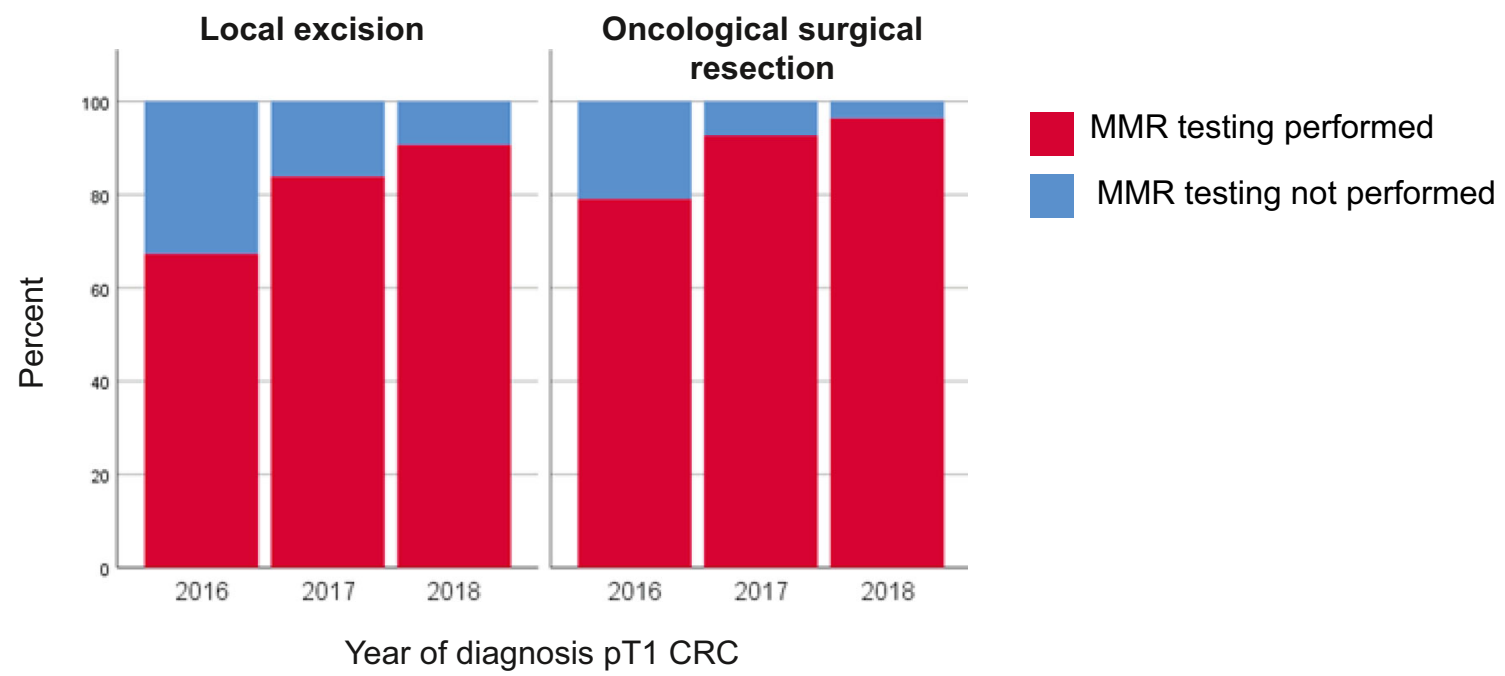

Fig. 3 Mismatch repair (MMR) testing in local excision $(n=2.560)$ and oncological surgical resection $(n=1.132)$ over time 
Table 2 Factors associated with mismatch repair (MMR) testing in $\mathrm{pT} 1$ colorectal cancer

\begin{tabular}{llll}
\hline & MMR testing performed $(n=3.095)$ & MMR testing not performed $(n=642)$ & $p$-value \\
\hline Gender & & & 0.13 \\
Male & $1855(82 \%)$ & $411(18 \%)$ & \\
Female & $1195(84 \%)$ & $231(16 \%)$ & $<0.01$ \\
Age at diagnosis & & & \\
$\leq 60$ years & $982(88 \%)$ & $140(12 \%)$ & $<0.01$ \\
$>61$ years & $2.068(80 \%)$ & $502(20 \%)$ & \\
Hospital & & $69(10 \%)$ & \\
Academic & $633(90 \%)$ & $573(19 \%)$ & \\
Non-academic & $2417(81 \%)$ & & \\
\hline
\end{tabular}

guideline or do not know on which material should be used for MMR testing) and limited knowledge.

MMR deficiency was detected in $6 \%$ of pT1 CRC in our retrospective cohort, while reported frequencies of MMR deficiency in sporadic CRC range from 15 to 20\% [26]. This relatively low frequency of MMR deficiency can be explained by our selection of pT1 cases diagnosed in participants of the Dutch screening program. Individuals from 55 to 75 years of age are invited to perform a biennial FIT. The frequency of MMR deficient CRC is associated with age; Lynch syndrome
Table 3 Characteristics of mismatch repair (MMR) proficient $(n=2.864)$ and MMR deficient $(n=186)$ pT1 colorectal cancer (CRC)

\begin{tabular}{|c|c|c|c|}
\hline & MMR proficient $(n=2864)$ & MMR deficient $(n=186)$ & $p$-value \\
\hline Gender & & & $<0.01$ \\
\hline Male & $1791(63 \%)$ & $64(34 \%)$ & \\
\hline Female & $1073(37 \%)$ & $122(66 \%)$ & \\
\hline Age categories & & & $<0.01$ \\
\hline $55-60$ years & $950(33 \%)$ & $32(17 \%)$ & \\
\hline $61-69$ years & $1914(67 \%)$ & $154(83 \%)$ & \\
\hline Location & & & $<0.01$ \\
\hline Proximal* & $365(13 \%)$ & $131(74 \%)$ & \\
\hline Distal & $2418(87 \%)$ & $47(26 \%)$ & \\
\hline Differentiation grade & & & $<0.01$ \\
\hline Well & $40(1 \%)$ & $3(2 \%)$ & \\
\hline Well/moderately & $2613(94 \%)$ & $155(89 \%)$ & \\
\hline Moderately & $59(2 \%)$ & $4(2 \%)$ & \\
\hline Poor/moderately & $66(3 \%)$ & $13(7 \%)$ & \\
\hline Unknown & 86 & 11 & \\
\hline Tumor budding & & & 0.45 \\
\hline No & $196(27 \%)$ & $10(27 \%)$ & \\
\hline Yes (not classified) & $86(12 \%)$ & $5(14 \%)$ & \\
\hline Low (Bd1) & $342(48 \%)$ & $20(54 \%)$ & \\
\hline Intermediate (Bd2) & $63(9 \%)$ & 0 & \\
\hline $\operatorname{High}(\mathrm{Bd} 3)$ & $33(4 \%)$ & $2(5 \%)$ & \\
\hline Unknown & 2144 & 149 & \\
\hline Lymphangioinvasion & & & 0.38 \\
\hline No & $2277(83 \%)$ & $149(87 \%)$ & \\
\hline Yes & $373(14 \%)$ & $17(10 \%)$ & \\
\hline Suspect & $86(3 \%)$ & $5(3 \%)$ & \\
\hline Unknown & 128 & 15 & \\
\hline Removal pT1 CRC & & & $<0.01$ \\
\hline Local excision & $1966(69 \%)$ & $82(44 \%)$ & \\
\hline Oncological surgical resection & $898(31 \%)$ & $104(56 \%)$ & \\
\hline
\end{tabular}

*Proximal location of pT1 CRC is when tumor is located in the cecum to splenic flexure 
is predominately detected in younger patients, while sporadic MMR deficient tumors are more often detected at an older age [27]. In our retrospective population, MMR deficient pT1 CRC were predominately detected at an older age $(83 \%$ at age 61-69 years), assuming that we mostly detected sporadic MMR deficient tumors. We probably miss the young patients with Lynch syndrome in our cohort.

A limitation of this study is the fact that there might be some missing data, since in less than $2 \%$ of cases, it was stated that the MMR status was still under investigation without any follow-up reports. Furthermore, we only included pT1 CRC diagnosed within the Dutch population-based screening program and therefore the minimum age at pT1 CRC diagnosis was 55 years. We do not have information about MMR testing adherence for pT1 CRC outside the screening program. The strength of this study is that we have data on a national level from 42/43 Dutch hospital laboratories, allowing differentiation between academic versus non-academic hospitals.

In conclusion, MMR testing was still only performed in $83 \%$ of the pT1 CRCs in the Netherlands between 2016 and 2018. However, in 2018, MMR status was evaluated in almost 92\% of the pT1 CRCs. By implementing MMR testing on the first endoscopic specimen obtained (either biopsy or local excision specimen), the uptake can easily be increased.

Acknowledgements We would like to acknowledge PALGA (Dutch Pathology Registry) for providing data. Dutch T1 CRC Working Group collaborators: Leon M.G. Moons (Department of Gastroenterology and Hepatology, University Medical Center Utrecht, Utrecht, the Netherlands); Sietze van Turenhout (Department of Gastroenterology and Hepatology, Noordwest Ziekenhuisgroep, Alkmaar, The Netherlands); Petur Snaebjornsson (Department of Pathology, Netherlands Cancer Institute, Amsterdam, The Netherlands); Michalda S. Dunker (Department of Surgery, Noordwest Ziekenhuisgroep, Alkmaar, The Netherlands).

Author contribution Conceptualization: BY, IN, AvB, and MvL; data curation: BY; formal analysis: BY; investigation: BY and MvL; methodology: BY, IN, AvB, and MvL; supervision: MvL; validation: BY and MvL; visualization: BY; writing-original draft: BY, IN, AvB, and MvL; writing-review and editing: KK, LM, SvT, PS, and MD

Data availability Data available upon reasonable request.

\section{Declarations}

Conflict of interest The authors declare no competing interests.

\section{References}

1. Moreira L, Balaguer F, Lindor N, de la Chapelle A, Hampel H, Aaltonen LA, Hopper JL, le Marchand L, Gallinger S, Newcomb PA, Haile R, Thibodeau SN, Gunawardena S, Jenkins MA, Buchanan DD, Potter JD, Baron JA, Ahnen DJ, Moreno V, Andreu M, Ponz de Leon M, Rustgi AK, Castells A, EPICOLON
Consortium (2012) Identification of Lynch syndrome among patients with colorectal cancer. JAMA 308:1555-1565

2. Newton K, Green K, Lalloo F, Evans DG, Hill J (2015) Colonoscopy screening compliance and outcomes in patients with Lynch syndrome. Color Dis 17:38-46

3. Moller P, Seppala T, Bernstein I et al (2017) Cancer incidence and survival in Lynch syndrome patients receiving colonoscopic and gynaecological surveillance: first report from the prospective Lynch syndrome database. Gut 66:464-472

4. Hampel H, Frankel WL, Martin E, Arnold M, Khanduja K, Kuebler P, Clendenning M, Sotamaa K, Prior T, Westman JA, Panescu J, Fix D, Lockman J, LaJeunesse J, Comeras I, de la Chapelle A (2008) Feasibility of screening for Lynch syndrome among patients with colorectal cancer. J Clin Oncol 26:5783-5788

5. Jarvinen HJ, Aarnio M, Mustonen $\mathrm{H}$ et al (2000) Controlled 15year trial on screening for colorectal cancer in families with hereditary nonpolyposis colorectal cancer. Gastroenterology 118:829834

6. Dutch Society of Clinical Genetics and Comprehensive Cancer Centre the Netherlands (2015) Dutch national guideline Hereditary Colorectal Cancer version 2.0. IKNL, Amsterdam

7. Vasen HF, Blanco I, Aktan-Collan K, Gopie JP, Alonso A, Aretz S, Bernstein I, Bertario L, Burn J, Capella G, Colas C, Engel C, Frayling IM, Genuardi M, Heinimann K, Hes FJ, Hodgson SV, Karagiannis JA, Lalloo F, Lindblom A, Mecklin JP, Møller P, Myrhoj T, Nagengast FM, Parc Y, Ponz de Leon M, RenkonenSinisalo L, Sampson JR, Stormorken A, Sijmons RH, Tejpar S, Thomas HJ, Rahner N, Wijnen JT, Järvinen HJ, Möslein G, Mallorca group (2013) Revised guidelines for the clinical management of Lynch syndrome (HNPCC): recommendations by a group of European experts. Gut 62:812-823

8. Toes-Zoutendijk E, Kooyker AI, Elferink MA, Spaander MCW, Dekker E, Koning HJ, Lemmens VE, van Leerdam M, LansdorpVogelaar I, LECO working group (2018) Stage distribution of screen-detected colorectal cancers in the Netherlands. Gut 67: 1745-1746

9. Casparie M, Tiebosch AT, Burger G, Blauwgeers H, van de Pol A, van Krieken J, Meijer GA (2007) Pathology databanking and biobanking in The Netherlands, a central role for PALGA, the nationwide histopathology and cytopathology data network and archive. Cell Oncol 29:19-24

10. van Rossum LG, van Rijn AF, Laheij RJ, van Oijen MG, Fockens P, van Krieken HH, Verbeek AL, Jansen JB, Dekker E (2008) Random comparison of guaiac and immunochemical fecal occult blood tests for colorectal cancer in a screening population. Gastroenterology 135:82-90

11. Hol L, van Leerdam ME, van Ballegooijen M, van Vuuren AJ, van Dekken H, Reijerink JCIY, van der Togt ACM, Habbema JDF, Kuipers EJ (2010) Screening for colorectal cancer: randomised trial comparing guaiac-based and immunochemical faecal occult blood testing and flexible sigmoidoscopy. Gut 59:62-68

12. Altieri DC (2001) Cytokinesis, apoptosis and survivin: three for tango? Cell Death Differ 8:4-5

13. van Lier MG, Wagner A, van Leerdam ME et al (2010) A review on the molecular diagnostics of Lynch syndrome: a central role for the pathology laboratory. J Cell Mol Med 14:181-197

14. Monahan KJ, Bradshaw N, Dolwani S, Desouza B, Dunlop MG, East JE, Ilyas M, Kaur A, Lalloo F, Latchford A, Rutter MD, Tomlinson I, Thomas HJW, Hill J, Hereditary CRC guidelines eDelphi consensus group (2020) Guidelines for the management of hereditary colorectal cancer from the British Society of Gastroenterology (BSG)/Association of Coloproctology of Great Britain and Ireland (ACPGBI)/United Kingdom Cancer Genetics Group (UKCGG). Gut 69:411-444 
15. Cavazza A, Radia C, Harlow C, Monahan KJ (2019) Experience of the implementation and outcomes of universal testing for Lynch syndrome in the United Kingdom. Color Dis 21:760-766

16. Evaluation of Genomic Applications in P, Prevention Working G (2009) Recommendations from the EGAPP Working Group: genetic testing strategies in newly diagnosed individuals with colorectal cancer aimed at reducing morbidity and mortality from Lynch syndrome in relatives. Genet Med 11:35-41

17. Syngal S, Brand RE, Church JM, Giardiello FM, Hampel HL, Burt RW, American College of Gastroenterology (2015) ACG clinical guideline: genetic testing and management of hereditary gastrointestinal cancer syndromes. Am J Gastroenterol 110:223-262 quiz 263

18. Leenen CH, Goverde A, de Bekker-Grob EW et al (2016) Costeffectiveness of routine screening for Lynch syndrome in colorectal cancer patients up to 70 years of age. Genet Med 18:966-973

19. Vos JR, Fakkert IE, Spruijt L, Willems RW, Langenveld S, Mensenkamp AR, Leter EM, Nagtegaal ID, Ligtenberg MJL, Hoogerbrugge N, FINAL Group (2020) Evaluation of yield and experiences of age-related molecular investigation for heritable and nonheritable causes of mismatch repair deficient colorectal cancer to identify Lynch syndrome. Int J Cancer 147:2150-2158

20. Vilkin A, Leibovici-Weissman Y, Halpern M et al (2015) Immunohistochemistry staining for mismatch repair proteins: the endoscopic biopsy material provides useful and coherent results. Hum Pathol 46:1705-1711

21. Kumarasinghe AP, de Boer B, Bateman AC, Kumarasinghe MP (2010) DNA mismatch repair enzyme immunohistochemistry in colorectal cancer: a comparison of biopsy and resection material. Pathology 42:414-420

22. Vasen HF, Watson P, Mecklin JP, Lynch HT (1999) New clinical criteria for hereditary nonpolyposis colorectal cancer (HNPCC, Lynch syndrome) proposed by the International Collaborative group on HNPCC. Gastroenterology 116:1453-1456

23. de Vos tot Nederveen Cappel WH, Buskens E, van Duijvendijk P et al (2003) Decision analysis in the surgical treatment of colorectal cancer due to a mismatch repair gene defect. Gut 52:1752-1755

24. Renkonen-Sinisalo L, Seppala TT, Jarvinen HJ, Mecklin JP (2017) Subtotal colectomy for colon cancer reduces the need for subsequent surgery in Lynch syndrome. Dis Colon Rectum 60:792-799

25. Panarelli NC, Somarathna T, Samowitz WS, Kornacki S, Sanders SA, Novelli MR, Shepherd NA, Yantiss RK (2016) Diagnostic challenges caused by Endoscopic Biopsy of Colonic Polyps: A Systematic Evaluation of Epithelial Misplacement with review of problematic polyps from the Bowel Cancer Screening Program, United Kingdom. Am J Surg Pathol 40:1075-1083

26. Poulogiannis G, Frayling IM, Arends MJ (2010) DNA mismatch repair deficiency in sporadic colorectal cancer and Lynch syndrome. Histopathology 56:167-179

27. van Lier MG, Leenen CH, Wagner A et al (2012) Yield of routine molecular analyses in colorectal cancer patients $</=70$ years to detect underlying Lynch syndrome. J Pathol 226:764-774

Publisher's note Springer Nature remains neutral with regard to jurisdictional claims in published maps and institutional affiliations. 